\title{
MS08-P09 | Structural Study For Recognition Of Ubiquitylated Histone H3 By DNA Methyltransferase
}

Arita, Kyohei (Graduate School of Medical Life Science, Yokohama City University, Yokohama, JPN); Ishiyama, Satoshi (Graduate School of Medical Life Science, Yokohama City University, Yokohama, JPN); Nishiyama, Atsuya (Division of Cancer Cell Biology, The Institute of Medical Science, The University of Tokyo, Tokyo, JPN); Defossez, Pierre-Antoine (Univ. Paris Diderot, Sorbonne Paris Cité, Epigenetics and Cell Fate, Paris, FRA); Nakanishi, Makoto (Division of Cancer Cell Biology, The Institute of Medical Science, The University of Tokyo, Tokyo, JPN)

Cytosine methylation in $\mathrm{CpG}$ dinucleotide plays an important role in gene silencing, genome imprinting, $\mathrm{X}$ chromosome inactivation and carcinogenesis. After DNA replication, DNA methylation patterns in somatic cells are faithfully maintained to upfold the cell identity. Two proteins are essential for the maintenance of DNA methylation; DNA methyltransferase DNMT1 and its recruiter UHRF1. After genome replication, UHRF1 SRA domain specifically recognize hemi-methylated DNA and subsequently catalyzes the multi-monoubiquitylation on histone $\mathrm{H} 3$ at K14, K18 and K23. DNMT1 RFTS domain recognizes the ubiquitylated histone H3, which recruits the DNMT1 on the DNA methylation sites.

Here, we present the structural basis for recognition of dual-monoubiquitylated histone H3 (ubiquitylated at K18 and K23) by DNMT1 RFTS domain. We show the sample preparation of ubiquitylated histone H3 analogs, detail of recognition of ubiquitylated histone $\mathrm{H} 3$, and activation of DNMT1 methyltransferase activity upon binding of the ubiquitylated histone H3. These data suggest that activation of DNMT1 at proper timing and location ensures the DNA methylation maintenance. Finally, we will discuss the recognition of triple monoubiquitylated histone $\mathrm{H} 3$ by DNMT1 RFTS. 